\title{
Intraoperative Electron Radiotherapy (IOERT) as an Alternative to Standard Whole Breast Irradiation: Only for Low-Risk Subgroups?
}

\author{
Sergio Maluta ${ }^{a}$ Stefano Dall'Oglio ${ }^{a}$ Donald A. Goer ${ }^{b}$ Nadia Marciaic \\ aDept. of Radiotherapy, University Hospital, Verona, Italy \\ bIntraop Medical Corporation, Sunnyvale, CA, USA \\ 'Dept. of Radiotherapy, Azienda Ospedaliera, Verona, Italy
}

\section{Keywords}

Breast cancer · Radiotherapy · Academic Review

\section{Summary}

Background: Intraoperative radiotherapy during breastconserving surgery is being studied as an alternative to 6 weeks of external beam radiotherapy (EBRT) for lowrisk women; it can be delivered using electrons (intraoperative electron radiotherapy, IOERT) or $50-\mathrm{kV}$ X-rays. Materials and Methods: We analyzed both single institutional and randomized studies involving single-fraction IOERT treatments. Rates for ipsilateral breast tumor recurrences, disease-specific survival, and overall survival were obtained. Results: IOERT had true 5-year recurrence rates of $<1.5 \%$ for ASTRO (American Society for Radiation Oncology) and ESTRO (European Society for Therapeutic Radiology and Oncology) suitable women, or for low-risk women as defined by the ELIOT trial. Women with ductal carcinoma in situ eligible for breastconserving therapy, older women that currently receive no EBRT, and younger women with favorable biology are additional potential candidates. Conclusion: ASTRO suitable and ESTRO good women for accelerated partial breast irradiation are low-risk groups. Higher-risk women with favorable biology might also be suitable candidates for IOERT, providing the tumor biology can be determined prior to surgery. For intraoperative radiotherapy using 50-kV X-rays, follow-up was too short to assess the effectiveness of the treatment.

\section{Introduction}

Intraoperative electron radiotherapy (IOERT) delivers radiation directly to the tumor bed during breast-conserving surgery (BCS) (concurrent approach), or, in some cases, it is delayed until after the pathological examination of the specimen (delayed approach). IOERT may be initially planned as a combined treatment with external radiotherapy (IOERT boost, i.e. IOERT followed by whole-breast irradiation, WBI), omitting the tumor bed radiation boost. In selected low-risk patients, IOERT could be delivered alone without any external irradiation as accelerated partial breast irradiation (IOERT APBI). If final pathology suggests adverse histological features, some patients initially planned for IOERT APBI will also receive 5 weeks of external beam radiotherapy (EBRT). Intraoperative radiotherapy is being studied using both 50-kV X-rays [1-4] and electrons (IOERT) [5-13]. When planned as a boost, intraoperative radiotherapy may be delivered to any patient that is BCS-eligible, with excellent results, irrespective of risk factors [14-20]. When delivered as APBI, data is emerging that low-risk patients are the ones most suitable for the technique. IOERT eliminates 1-6 weeks of radiotherapy, making it not only cost-effective but convenient for the patient. This is true for patients unable to have postoperative radiation or living at locations where radiotherapy is not available. IOERT is a way of administering radiotherapy at low cost, reducing the risk of a complete lack of irradiation. It is applied directly to the tumor bed using visual guidance, eliminating the possibility of a geometric miss of the tumor bed, which may still be possible with EBRT despite improved radiotherapy imaging approaches. When used as APBI, IOERT avoids expensive and time-consuming systems such as image-guided radiotherapy or intensity-modulated radiotherapy, or breathing control. Intraoperative radiotherapy reduces skin dose, and, when delivered as APBI, irradiates less healthy tissue than conventional EBRT approaches. The delivery of radiation at the time of surgery (in the concurrent approach) has improved radiobiological benefits as it starts irradiation at a time when the residual tumor cells are more rapidly proliferating. Finally, it has logistical benefits in terms of less treatment time, thereby reducing patients' transporta-

\section{KARGER}

Fax +497614520714

Information@Karger.com

www.karger.com (c) 2014 S. Karger GmbH, Freiburg

$1661-3791 / 14 / 0092-0102 \$ 39.50 / 0$

Accessible online at:

www.karger.com/brc
Sergio Maluta, MD

Dept. of Radiotherapy

University Hospital Verona

and Hyperthermia Unit of Medical Center Serena, Padua

Piazzale Stefani 1, 37126 Verona, Italy

sergiomaluta@yahoo.it 
tion costs, and psychological benefits by reducing patients' stress and anxiety caused by waiting for EBRT treatment to begin. These advantages compared to postoperative radiotherapy after BCS are the reason why there is interest in expanding intraoperative radiotherapy use to women other than those at low risk.

\section{Materials and Methods}

We limited our review to the intraoperative radiotherapy APBI techniques, and found 17 peer-reviewed articles with adequate patient volumes to utilize [1-13, 15, 21-23]. As many women today are on hormonal therapy which is known to delay recurrences, we decided to examine only studies with actual follow-ups of 5 years; studies with intraoperative highdose-rate brachytherapy $[15,21]$ were not taken into consideration, as this is only being used in 1 center and the APBI volume irradiated is inadequate. The TARGIT-A studies $[1,4,18]$, while illustrating several useful points, in our opinion had insufficient follow-up to meet our selection criteria.

\section{Results}

Veronesi et al. [24] randomized 1,305 women age $>48$ years with tumors $<2.5 \mathrm{~cm}$ to either an IOERT APBI dose of $21 \mathrm{~Gy}$ prescribed to the $90 \%$ point (ELIOT trial), or 5 weeks of WBI with a 10-Gy EBRT boost. Both intraductal carcinomas and lobular carcinomas were included. All patients received wide excision (quandrantectomy) surgery. ELIOT patients had a protective metallic disk inserted below the mammary gland to protect the chest wall. The trial closed in December 2007, and analysis began 5 years after the last patient was accrued. The median follow-up was 5.9 years for the EBRT patients and 5.5 years for the IOERT patients. ELIOT and EBRT patients achieved 5-year recurrence rates of 4.4 and $0.4 \%$, respectively $(\mathrm{p}<0.0001)$. When analyzing for risk of relapse, the 5-year ipsilateral breast tumor recurrence (IBTR) rate exceeded $10 \%$ for patients with tumors $>2 \mathrm{~cm}(10 / 83$ $=10.9 \%), 4$ or more positive nodes $(4 / 31=15.0 \%)$, poorly differentiated tumors, i.e. G3 $(15 / 129=11.9 \%)$, estrogen re- ceptor-negative tumors $(8 / 63=14.9 \%)$, or triple-negative disease $(7 / 43=18.9 \%)$. Patients with a high proliferative index, i.e. Ki-67 > 20\%, also trended to have a high IBTR rate $(22 / 244=9.1 \%)$, but this was not used in their risk factor analysis since it did not reach the imposed $10 \%$ threshold. The 5-year IBTR rate was $11.3 \%$ for the 199 (30.6\%) women with 1 or more of these risk factors, versus only $1.5 \%$ for the remaining $452(69.4 \%)$ women who had none of these factors.

A non-randomized study of 1,822 non-selected patients treated with the ELIOT technique showed a 5-year IBTR rate of $6 \%$. When analyzed according to ASTRO (American Society for Radiation Oncology) and ESTRO (European Society for Therapeutic Radiology and Oncology) guidelines for patients 'suitable' or 'good' for APBI treatment, the 5-year IBTR rates were only 1.5 and $1.9 \%$, respectively $[10,11]$ (table 1). These APBI favorable groups also did significantly better than the less favorable APBI category patients in terms of distant metastasis, any breast-related events, cause-specific survival, and overall survival. There was no difference in regional lymph node failure among these categories of patients. Tumor grade, however, though not a selection criterion by either ASTRO or ESTRO, was a significant factor for IBTR, regional node failure, and distant metastasis. While neither ASTRO nor ESTRO consider IOERT in their selection criteria for suitable or good APBI patients, application of these guidelines to the 1,822 out-trial patients suggests that they might also form a reasonable basis for selection of IOERT APBI patients. The $1.5 \%$ recurrence rate for ASTRO suitable women in this study is consistent with the recurrence rate reported for the $22 \%$ of ASTRO suitable women in the ELIOT randomized trial, but the higher than expected recurrence rate of $6 \%$ for the total cohort of 1,822 patients led the authors to question whether the tumor bed in their study was properly covered in terms of volume and dosage. IOERT is delivered to the tumor bed under direct visualization, so a geometric miss as might occur with EBRT is not possible. Their dose of 21 Gy should have been sufficient to sterilize microscopic disease surrounding the tumor. However, the

Table 1. ELIOT study results according to ASTRO and ESTRO guidelines; analysis of 1,822 patients treated with ELIOT off-trial produced very low local recurrence rates for ASTRO suitable and ESTRO good patients, and luminal A patients do well with APBI irrespective of the ASTRO/ESTRO category; age, tumor size, and biology remain independent prognosticators for recurrence with IOERT APBI

\begin{tabular}{|c|c|c|c|c|c|c|}
\hline & $\begin{array}{l}\text { Patients, } \\
\mathrm{n}(\%)\end{array}$ & $\begin{array}{l}\text { Local relapse, } \\
\mathrm{n}\end{array}$ & $\begin{array}{l}5 \text {-year rate, } \\
\%\end{array}$ & $\begin{array}{l}\text { Luminal A, } \\
\mathrm{n}\end{array}$ & $\begin{array}{l}\text { Loco-regional } \\
\text { relapse, } \mathrm{n}\end{array}$ & $\begin{array}{l}\text { 5-year rate, } \\
\%\end{array}$ \\
\hline \multicolumn{7}{|c|}{ ASTRO guidelines } \\
\hline All & 1,822 & 76 & 6.0 & 648 & 8 & 1.7 \\
\hline Suitable & 295 (16) & 3 & 1.5 & 118 & 2 & 2.3 \\
\hline Cautionary & $690(38)$ & 21 & 4.4 & 271 & 3 & 1.6 \\
\hline Unsuitable & $812(45)$ & 50 & 8.8 & 251 & 3 & 1.6 \\
\hline Not accessible & $25(1)$ & 2 & 9.9 & 8 & 0 & - \\
\hline \multicolumn{7}{|l|}{ ESTRO guidelines } \\
\hline All & 1,822 & 76 & 6.0 & 648 & 8 & 1.7 \\
\hline Good & $572(31)$ & 7 & 1.9 & 206 & 0 & 0.0 \\
\hline Possible & $268(15)$ & 12 & 7.1 & 129 & 2 & 2.5 \\
\hline Unsuitable & $965(53)$ & 56 & 7.8 & 306 & 6 & 2.4 \\
\hline Not accessible & $17(1)$ & 1 & 6.6 & 8 & 0 & - \\
\hline
\end{tabular}


Table 2. University of Verona recurrences after APBI compared to ASTRO/ESTRO low-risk women

\begin{tabular}{|c|c|c|c|c|c|c|}
\hline Factor & Patient 1 & Patient 2 & Patient 3 & Patient 4 & ASTRO suitable & ESTRO good \\
\hline Age, years & 55 & 62 & 68 & 75 & $\geq 60$ & $\geq 50$ \\
\hline Histology & IDC & IDC & IDC & IDC & $\mathrm{IDC}^{\mathrm{b}}$ & $\mathrm{IDC}^{\mathrm{b}}$ \\
\hline Tumor size, $\mathrm{cm}$ & 2.0 & 2.8 & 1.5 & 1.8 & $\leq 2.0$ & $\leq 3.0$ \\
\hline Grade & G2 & G2 & G3 & G3 & any & any \\
\hline Nodal status & $\mathrm{pN} 0$ & $\mathrm{pN} 0$ & $\mathrm{pN} 0$ & pN0 & pN0 & pN0 \\
\hline ER status & positive & positive & positive & negative & positive & any \\
\hline PR status & positive & positive & positive & negative & positive & any \\
\hline HER2 status & negative & unknown & negative & unknown & NS & NS \\
\hline LVI status & absent & absent & absent & present & not allowed & not allowed \\
\hline Margin status & negative & negative & negative & negative & negative & negative \\
\hline Adjuvant HT/CT & $\mathrm{HT}$ & $\mathrm{HT}$ & $\mathrm{HT}$ & none $^{\mathrm{a}}$ & & \\
\hline Time to relapse, months & 28 & 36 & 40 & 60 & & \\
\hline Salvage & mastectomy & mastectomy & mastectomy & mastectomy & & \\
\hline
\end{tabular}

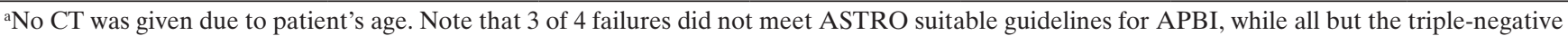
met the ESTRO good guidelines for APBI. If G3 and tumors $>2.0 \mathrm{~cm}$ are excluded, there is only 1 recurrence in the University of Verona cohort with a mean follow-up of 51 months.

bOther low-risk histologies also permitted.

IDC = Invasive ductal carcinoma; ER = estrogen receptor; PR = progesterone receptor; LVI = lymphovascular invasion; HT = hormonal therapy;

$\mathrm{CT}=$ chemotherapy; NS $=$ not significant.

median applicator size of $4 \mathrm{~cm}$ may have been too small to adequately cover larger tumors. When using a 4-cm applicator, even though the IOERT surgical preparation brings almost $2 \mathrm{~cm}$ of surrounding tissue within the range of the applicator, only about $1.5 \mathrm{~cm}$ is irradiated to the prescription dose of $90 \%$. Tissues $4-5 \mathrm{~mm}$ from the walls of the applicator receive less radiation. To assure uniform coverage of the tissues at risk, the applicator should be chosen 1.5-2 cm larger than the maximum tumor dimension. The authors acknowledge that tissues at the periphery of these smaller applicators might have been inadequately irradiated.

The University of Verona study of IOERT APBI in 226 women began in 2006 [8]. Patient selection criteria were restricted to only very low-risk patients, essentially ASTRO good or ESTRO suitable. All patients had quandrantectomy and axillary node management, including sentinel node evaluation and complete axillary node dissection (CAND) for positive sentinel nodes, but the radiation technique was modified from the ELIOT approach. The prescribed dose was reduced by about $10 \%$, so the maximum dose to the gland was $21 \mathrm{~Gy}$ with the energy selected to assure that the entire gland was covered to at least the $80 \%$ dose line. Verona used a $10-\mathrm{mm}$ thick acrylic disk to protect the chest wall, rather than the metallic plates used with ELIOT. The use of the acrylic disk eliminated any dose uncertainties from backscatter caused by metallic disks. Acrylic disks were possible in this study because patients were treated to the $80 \%$ dose line; hence, most patients $(95.5 \%)$ were able to be treated with electron energies of $6 \mathrm{MeV}$ or less. At this energy level, the transmitted dose is at the most 3.2 Gy which is of no consequence clinically. $50(22.1 \%)$ patients that had a positive sentinel node biopsy (SNB) and underwent CAND, 38 had 1 positive lymph node and 12 had 2, with all receiving IOERT. 16 (7.1\%) patients were shown to have positive margins on final pathology and underwent re-excision. An additional 17 (7.5\%) patients had close margins $(<2 \mathrm{~mm}$ clearance $)$, but received no further treatment. No additional radiation was given to either patients with positive nodes or positive margins. At the time of the initial report, only 1 patient had recurred with a mean follow-up of 46 months. The Verona results were updated at the 2012 ASCO Breast Meeting and the SABCS Meeting, and today, with a median follow-up of 62 months, there have been 4 recurrences (table 2). All recurrences were salvaged with mastectomy. All patients in the study are alive and diseasefree. Importantly, in contrast to the ELIOT studies, the applicator size chosen was approximately $2 \mathrm{~cm}$ greater in diameter than the largest tumor dimension. The median applicator size used was $6 \mathrm{~cm}$, assuring good coverage of the tumor bed, with $87 \%$ of the field sizes $>5 \mathrm{~cm}$ and $31 \%>6 \mathrm{~cm}$.

\section{Younger Women}

It is clear from tables 1 and 2 that the standard low-risk groups for IOERT APBI should be ASTRO suitable or ESTRO good. However, it may also be possible to expand this group to women with a more favorable biology such as luminal A, which can include younger women. If younger women are included, it would be advisable to ascertain that they have none of the high-risk factors found in the ELIOT trial.

\section{Ductal Carcinoma in Situ}

Despite the high incidence of ductal carcinoma in situ (DCIS) [25] and a great number of trials, the definitive treatment is still controversial: mastectomy vs. BCS, sentinel lymph node or not, WBI vs. APBI. These controversies are due to the heterogeneity of DCIS and limited published clinical trials. There is no data on DCIS left untreated, but a review of autopsy records showed that $9-15 \%$ of women have undetected DCIS at death [26]. DCIS is usually treated like early-stage invasive breast cancer: BCS and radiotherapy or mastectomy $+/-$ hormonal therapy. Overall survival is high, $96-98 \%$ at 10 years [27]. 4 prospective randomized 
trials (NSABP B-17 [28], EORTC 10853 [29], SweDCIS [30], UK/ANZ [31]) showed a $50 \%$ reduction in local failure when radiotherapy was added after surgery, and even though none of the trials showed differences in overall survival, they were unable to identify any subset of patients who did not benefit from radiotherapy. Vicini et al. [32] analyzed outcomes in 300 women with DCIS, who had APBI as part of the American Society of Breast Surgeons Mammosite Registry trial. The IBTR rate was $2.6 \%$ at 5 years, while cause-specific survival was $99.5 \%$ and overall survival $96.4 \%$. When the ASTRO cautionary DCIS group (125 patients with pure DCIS $<3 \mathrm{~cm}$ ) was compared to the invasive suitable/cautionary group, no difference in IBTR was noted with significant improvement in distant metastasis ( 0 vs. $2.5 \%$ ), disease-free survival (98.5 vs. $94.4 \%$ ), and overall survival (95.7 vs. 90\%). Even though DCIS patients are usually excluded from IOERT studies and fall outside of the ASTRO suitable and ESTRO good categories for APBI, this encouraging result for APBI should be tested within a controlled trial to assess whether IOERT has a role in women who require radiation after BCS for DCIS.

\section{Older Women}

Older women would appear to be good candidates for IOERT, since most are likely to be ASTRO suitable or ESTRO good for APBI. However, older age can often deter women from receiving radiation treatment. Studies have shown that estrogen receptor-positive women older than 70 years and on hormone therapy have equivalent survival after BCS whether or not they receive EBRT, despite the fact that those who do not receive EBRT have an absolute increase in local failure of about $7 \%$ at 5 years [33-35]. Since completion of radiotherapy also decreases from 84 to $42 \%$ when the distance to a radiation center increases from 25 to 100 miles or more, older women living far from a radiation center are at especially high risk of not receiving the optimal treatment for their breast cancer. IOERT can potentially reduce the number of salvage mastectomies in these older women by better controlling the disease with a single dose of IOERT during the initial BCS.

\section{Discussion}

IOERT APBI is still in the early stages of development, but it is already clear that it is not suitable for all women, and that patient selection and careful attention to technique are critical to proper implementation. IOERT APBI appears to have a subset of low-risk women for whom IOERT will be effective. The University of Verona data (table 2) shows a cumulative IBTR incidence of only $1.8 \%$, with a follow-up of more than 5 years. Compared to ASTRO suitable, ESTRO good, or ELIOT low-risk patients, the 5-year cumulative recurrence rates for the Verona patients were $0.4,1.3$ and $0.4 \%$, respectively. Despite the excellent results demonstrated for these low-risk women, IOERT APBI has been criticized because the final margin and nodal status is not known at the time of IOERT delivery. At the University of Verona, 16 (7.1\%) patients were shown to have positive margins on final pathology and underwent re-excision. An additional 17 $(7.5 \%)$ patients had close margins $(<2 \mathrm{~mm}$ clearance $)$ but received no further treatment. $50(22.1 \%)$ patients had a positive SNB and underwent CAND. Of these 50 patients, $38 \mathrm{had}$ 1 positive lymph node and 12 had 2 . No additional radiation was given to either patients with positive nodes or positive margins. None of the patients who recurred had positive or close margins or positive nodes. The ELIOT trial also found that margin positivity or 1-3 positive nodes were not high-risk factors with IOERT. Perhaps with single-dose IOERT, positive margins are not as much of a concern as they are in conventionally treated patients. Jobsen et al. [36] found that for women over 40 years of age, margin positivity had no influence on recurrence. The radiation technique for IOERT APBI brings all of the margins to the center of the radiation field such that they receive at least $18 \mathrm{~Gy}$ in a single exposure. This is likely to be an adequate dose to sterilize any microscopic residual disease in the margins.

Extending IOERT APBI to younger women with favorable biology (table 1) could be possible, and is already being tested at a number of centers. There is also no reason why carefully screened and selected DCIS patients should not benefit from IOERT, either as a boost or as APBI. And finally, IOERT APBI would appear to be an appropriate treatment for older women (> 70 years) who are not currently receiving radiation. IOERT APBI has the potential of reducing recurrences and thus avoiding salvage mastectomies in this elderly population.

A number of questions concerning IOERT APBI remain to be answered: i) Will the lesser surgeries used outside of Italy produce equivalent results to quandrantectomy?; ii) Is 21 Gy to the $90 \%$ dose level the optimal dose, or can a lower dose such as used in Verona be used?; iii) What is the best way to handle positive margins on final pathology? Ignore them? Re-excise them? Do these patients require a mastectomy?; iv) What is the best way to handle positive nodes (positive nodes are not permissible in other post-op APBI trials)?; and v) With IOERT, are 1-2 positive nodes after SNB and CAND an important factor in ipsilateral recurrence, since the radiation treatment to the tissue at risk for local recurrence has already been delivered? Further studies will be needed to answer these important questions.

\section{Conclusion}

ASTRO suitable and ESTRO good women for APBI are low-risk groups that appear to do well with IOERT if there is proper irradiation of the tissue volumes at risk. Higher-risk 
women with favorable biology might also be suitable candidates, provided the biology can be determined prior to surgery. Because IOERT APBI is still in the early stages of its clinical development, it is advisable that all IOERT APBI patients be treated according to protocol.

\section{Disclosure Statement}

D. Goer is chief scientist at Intraop Medical. All the authors declare on their own responsibility that they have no conflicts of interest of any kind.

\section{References}

1 Vaidya JS, Joseph DJ, Tobias JS, et al.: Targeted intraoperative radiotherapy versus whole breast radiotherapy for breast cancer (TARGIT-A trial): an international, prospective, randomized, non-inferiority phase 3 trial. Lancet 2010;376:91-102.

2 Kraus-Tiefenbacher U, Scheda A, Steil V, et al.: Intraoperative radiotherapy (IOERT) for breast cancer using the intrabeam system. Tumori 2005; 91:339-345.

3 Tuschy B, Berlit S, Romero S, et al.: Clinical aspects of intraoperative radiotherapy in early breast cancer: short term complications after IOERT in women treated with low energy x-rays. Radiat Oncol 2013;22:8-95.

4 Vaidya, JS, Wenz F, Bulsara M, et al.: Risk-adapted targeted intraoperative radiotherapy versus whole-breast radiotherapy for breast cancer: 5-year results for local control and overall survival from the TARGIT-A randomized trial. Lancet 2014; 383:603-613.

$\checkmark 5$ Veronesi U, Orecchia R, Luini A, et al.: Full-dose intraoperative radiotherapy with electrons during breast-conserving surgery: experience with 590 cases. Ann Surg 2005;242:101-106.

6 Ivaldi GB, Leonardi MC, Orecchia R, et al.: Preliminary results of electrons intraoperative therapy boost and hypofractionated external beam radiotherapy after breast-conserving surgery in premenopausal women. Int J Radiat Oncol Biol Phys 2008;72:485-493.

7 Lemanski C, Azria D, Gourgon-Bourgade S, et al.: Intraoperative radiotherapy in early-stage breast cancer: results of the Montpellier phase II trial. Int J Radiat Oncol Biol Phys 2010;76:698-703.

$\checkmark 8$ Maluta S, Dall'Oglio S, Marciai N, et al.: Accelerated partial breast irradiation using only intraoperative electron radiation therapy in early stage breast cancer. Int J Radiat Oncol Biol Phys 2012; $84: 145-152$.

$\checkmark 9$ Leonardi MC, Ivaldi GB, Santoro L, et al.: Longterm side effects and cosmetic outcome in a pool of breast cancer patients treated with intraoperative radiotherapy with electrons as sole treatment. Tumori 2012;98:324-330

10 Leonardi MC, Maisonneuve P, Mastropasqua MG et al.: How do the ASTRO consensus statement guidelines for the application of accelerated partial breast irradiation fit intraoperative radiotherapy? A retrospective analysis of patients treated at the European Institute of Oncology. Int J Radiat Oncol Biol Phys 2012;83:806-813.

-11 Leonardi MC, Maisonneuve P, Mastropasqua MG, et al.: Accelerated partial breast irradiation with intraoperative electrons: using GEC-ESTRO recommendations as guidance for patient selection. Radiother Oncol 2013;106:21-27.

12 Rampinelli C, Bellomi M, Ivaldi GB, et al.: Assessment of pulmonary fibrosis after radiotherapy (RT) in breast conserving surgery: comparison between conventional external beam RT (EBRT) and intraoperative RT with electrons (ELIOT). Technol Cancer Res Treat 2011;10:323-329.

13 Dall'Oglio S, Maluta S, Marciai N, et al.: Intraoperative electron radiotherapy in early-stage breast cancer: a mono-institutional experience. Poster presentation SABCS 2012; abstr P3-13-07.

14 Sedlmayer F, Fastner G, Merz F, et al.: IOERT with electrons as boost strategy during breast conserving therapy in limited stage breast cancer: results of an ISIOERT pooled analysis. Strahlenther Onkol 2007;183:32-34.

15 Beal K, McCormick B, Zelefsky MJ, et al.: Singlefraction intraoperative radiotherapy for breast cancer: early cosmetic results. Int J Radiat Oncol Biol Phys 2007;69:19-24.

16 Fastner G, Sedlmayer F, Merz F, et al.: IOERT with electrons as boost strategy during breast conserving therapy in limited stage breast cancer: long term results of an ISIOERT pooled analysis. Radiother Oncol 2013;108:279-286.

17 Lemanski C, Azria D, Thezenas S, et al.: Intraoperative radiotherapy given as a boost for early breast cancer: long-term clinical and cosmetic results. Int J Radiat Oncol Biol Phys 2006;64:14101415.

18 Vaidya JS, Baum M, Tobias JS, et al.: Long-term results of targeted intraoperative radiotherapy (TARGIT) boost during breast-conserving surgery. Int J Radiat Oncol Biol Phys 2011;81:10911097.

19 Wenz, F, Welzel G, Keller A, et al.: Early initiation of external beam radiotherapy (EBRT) may increase the risk of long-term toxicity in patients undergoing intraoperative radiotherapy (IOERT) as a boost for breast cancer. Breast 2008;17:617622 .

20 Wenz F, Welzel G, Blank E, et al.: Intraoperative radiotherapy as a boost during breast-conserving surgery using low-kilovoltage $\mathrm{x}$-rays: the first 5 years of experience with a novel device. Int $\mathrm{J}$ Radiat Oncol Biol Phys 2010;77:1309-1314.

21 Beal K Sacchini V, Zelefsky M, et al.: Five year update on intraoperative radiation therapy for breast cancer. Int $\mathrm{J}$ Radiat Oncol Biol Phys Suppl to Annual Meeting 2011;S241-S242(abstr 2061).

22 Kimple RJ, Klauber-DeMore N, Kuzmiak CM, et al.: Local control following single-dose intraoperative radiotherapy prior to surgical excision of earlystage breast cancer. Ann Surg Oncol 2011;18:939945.

23 Kimple RJ, Klauber-DeMore N, Kuzmiak CM, et al.: Cosmetic outcomes for accelerated partial breast irradiation before surgical excision of early stage breast cancer using single-dose intraoperative radiotherapy. Int J Radiat Oncol Biol Phys 2011; 79:400-407.

24 Veronesi U, Orecchia R, Maisonneuve P, et al.: Intraoperative radiotherapy versus external radiotherapy for early breast cancer (ELIOT): a ran- domized controlled equivalence trial. Lancet Oncol 2013;14:1269-1277.

25 Sprague BL, Trentham-Dietz A: Prevalence of breast carcinoma in situ in the United States. JAMA 2009;302:846-848.

26 Welch HG, Black WC: Using autopsy series to estimate the disease reservoir for ductal carcinoma in situ of the breast: how much more breast cancer can we find? Ann Intern Med 1997;127:1023-1028.

27 Allegra CJ, Aberle DR, Ganschow P, et al.: National Institute of Health State-of-the-Science Conference statement: Diagnosis and Management of Ductal Carcinoma in Situ, September 22-24, 2009. J Natl Cancer Inst 2010;102:161-169.

28 Fisher B, Costantino J, Redmond C, et al. Lumpectomy compared with lumpectomy and radiation therapy for the treatment of intraductal breast cancer. N Engl J Med 1993;328:1581-1586.

29 Julien JP, Bijker N, Fentiman IS, et al.: Radiotherapy in breast-conserving treatment for ductal carcinoma in situ: first results of the EORTC randomized phase III trial 10853. EORTC Breast Cancer Cooperative Group and EORTC Radiotherapy Group. Lancet 2000;355:528-533.

30 Emdin SO, Granstrand B, Ringberg A, et al.: SweDCIS: radiotherapy after sector resection for ductal carcinoma in situ of the breast. Results of a randomised trial in a population offered mammography screening. Acta Oncol 2006;45:536543.

31 Houghton J, George WD, Cuzick J, et al.: Radiotherapy and tamoxifen in women with completely excised ductal carcinoma in situ of the breast in the UK, Australia, and New Zealand: randomized controlled trial. Lancet 2003;362:95-102.

32 Vicini F, Shah C, Wilkinson J, et al.: Should ductal carcinoma in situ (DCIS) be removed from the ASTRO consensus panel cautionary group for off-protocol use of accelerated partial breast irradiation? A pooled analysis of outcomes for $300 \mathrm{pa}$ tients with DCIS treated with APBI. Ann Surg Oncol 2013;20:1275-1281.

33 Fyles AW, McCready DR, Manchul LA, et al.: Tamoxifen with or without breast irradiation in women 50 years of age or older with early breast cancer. N Engl J Med 2004;351:963-970.

34 Blamey RW, Bates T, Chetty U, et al.: Radiotherapy or tamoxifen after conserving surgery for breast cancers of excellent prognosis: British Association of Surgical Oncology (BASO) II trial. Eur J Cancer 2013;49:2294-2302.

35 Hughes KS, Schnaper LA, Berry D, et al.: Lumpectomy plus tamoxifen with or without irradiation in women 70 years of age or older with early breast cancer. N Engl J Med 2004;351:971-977.

36 Jobsen JJ, van der Palen J, Ong F, Meerwaldt JH: The value of a positive margin for invasive carcinoma in breast-conservative treatment in relation to local recurrence is limited to young women only. Int J Radiat Oncol Biol Phys 2003;57:724-731. 